\title{
What is new on molecular characteristics of Avian metapneumovirus strains circulating in Europe?
}

\author{
Giulia Mescolini ${ }^{1}$, Caterina Lupini ${ }^{1}$, Giovanni Franzo ${ }^{2}$, Giulia Quaglia ${ }^{1}$, Matteo Legnardi ${ }^{3}$, \\ Mattia Cecchinato ${ }^{4}$, Claudia Tucciarone ${ }^{3}$, Angela Blanco ${ }^{5}$, Vincent Turblin ${ }^{6}$, Mar Biarnés \\ Suñé $^{5}$, Fabrizio Tatone ${ }^{7}$, Marco Falchieri ${ }^{2}$, and Elena Catelli ${ }^{1}$ \\ ${ }^{1}$ University of Bologna \\ ${ }^{2}$ Affiliation not available \\ ${ }^{3}$ Universita degli Studi di Padova - Campus di Agripolis \\ ${ }^{4}$ university of Padova \\ ${ }^{5}$ CESAC - Centre de Sanitat Avícola de Catalunya i Aragó \\ ${ }^{6} \mathrm{MC}$ VET Conseil - RESEAU CRISTAL \\ ${ }^{7}$ RESALAB - RESEAU CRISTAL
}

May 19, 2020

\begin{abstract}
In the present study one hundred and sixteen partial G gene sequences of Avian metapneumovirus (aMPV) subtype B, obtained during routine diagnostics in different European Countries in the last few years (2014-2019), were analysed by sequence and phylogenetic analyses in order to draw an updated picture of the molecular characteristics of circulating strains. Nucleotide sequences were compared with other sequences of European and extra-European aMPV-Bs collected prior to that period or retrieved from GenBank. Phylogenetic relationships among the aMPV-B strains, reconstructed using the Maximum Likelihood method implemented in MEGA X, demonstrated that aMPV-B has evolved in Europe from its first appearance, frequently displaying a clear relation with the geographic area of detection. The $40 \%$ of aMPV-B viruses analysed were classified as vaccine-derived strains, being phylogenetically related, and showing high nucleotide identity with live commercial vaccine strains licensed in Europe. The remaining $60 \%$ were classified as field strains since they clustered separately and showed a low nucleotide identity with vaccines and vaccine-derived strains. The phylogenetic tree showed that the virus has continued to evolve from its first appearance in the ' 80 s since more recently detected strains belonged to clades phylogenetically distant from the older strains. Unlike vaccine-derived strains, field strains tended to cluster according to their geographic origin and irrespective of the host species where the viruses had been detected. In conclusion, the molecular characterization of aMPV-B and the differentiation between vaccines and field strains through G gene sequence analysis can be a useful tool towards correct diagnosis and should be routinely applied in order to better address the control strategies.
\end{abstract}

What is new on molecular characteristics of Avian metapneumovirus strains circulating in Europe?

Molecular epidemiology of aMPV-B in Europe

Giulia Mescolini ${ }^{1}$, Caterina Lupini ${ }^{1 *}$, Giovanni Franzo ${ }^{2}$, Giulia Quaglia ${ }^{1}$, Matteo Legnardi ${ }^{2}$, Mattia Cecchinato $^{2}$, Claudia Maria Tucciarone ${ }^{2}$, Angela Blanco ${ }^{3}$, Vincent Turblin ${ }^{4}$, Mar Biarnés ${ }^{3}$, Fabrizio Tatone ${ }^{5}$, Marco Falchieri ${ }^{6}$, Elena Catelli ${ }^{1}$

${ }^{1}$ Department of Veterinary Medical Sciences, University of Bologna, Ozzano dell'Emilia (BO), Italy 
${ }^{2}$ Department of Animal Medicine, Production and Health, University of Padua, Legnaro (PD), Italy

${ }^{3}$ CESAC - Centre de Sanitat Avícola de Catalunya i Aragó, Reus, Spain.

${ }^{4}$ MC VET Conseil - RESEAU CRISTAL, Sablé sur Sarthe, France

${ }^{5}$ RESALAB - RESEAU CRISTAL, Les Herbiers, France

${ }^{6}$ Field Veterinarian, United Kingdom

*Corresponding author: Caterina Lupini, Department of Veterinary Medical Sciences -University of Bologna, Via Tolara di Sopra, 50; 40064 - Ozzano dell'Emilia (BO) Italy; Phone +39 051 2097559; email:caterina.lupini@unibo.it

\section{Summary}

In the present study one hundred and sixteen partial G gene sequences of Avian metapneumovirus (aMPV) subtype B, obtained during routine diagnostics in different European Countries in the last few years (20142019), were analysed by sequence and phylogenetic analyses in order to draw an updated picture of the molecular characteristics of circulating strains. Nucleotide sequences were compared with other sequences of European and extra-European aMPV-Bs collected prior to that period or retrieved from GenBank. Phylogenetic relationships among the aMPV-B strains, reconstructed using the Maximum Likelihood method implemented in MEGA X, demonstrated that aMPV-B has evolved in Europe from its first appearance, frequently displaying a clear relation with the geographic area of detection. The $40 \%$ of aMPV-B viruses analysed were classified as vaccine-derived strains, being phylogenetically related, and showing high nucleotide identity with live commercial vaccine strains licensed in Europe. The remaining $60 \%$ were classified as field strains since they clustered separately and showed a low nucleotide identity with vaccines and vaccinederived strains. The phylogenetic tree showed that the virus has continued to evolve from its first appearance in the ' 80 s since more recently detected strains belonged to clades phylogenetically distant from the older strains. Unlike vaccine-derived strains, field strains tended to cluster according to their geographic origin and irrespective of the host species where the viruses had been detected. In conclusion, the molecular characterization of aMPV-B and the differentiation between vaccines and field strains through $\mathrm{G}$ gene sequence analysis can be a useful tool towards correct diagnosis and should be routinely applied in order to better address the control strategies.

\section{Key words: Avian metapneumovirus, subtype B, Europe, molecular characterization}

\section{Introduction}

Avian metapneumovirus (aMPV) infections in Europe have been an issue since its first appearance both in turkeys, chickens and in other minor species (Toquin, Bayon-Auboyer, Eterradossi, \& Jestin, 1999; Catelli et al., 2001; Cecchinato, Ferreira, Munir, \& Catelli, 2017; Cecchinato et al., 2018), causing economic losses mainly due to respiratory or reproductive problems (Cecchinato et al., 2012), often exacerbated by secondary bacterial infections (Giovanardi et al., 2014).

First aMPV isolations in Europe date back to the second half of the 1980s, when Turkey Rhinotracheitis (TRT) outbreaks appeared in the United Kingdom (McDougall and Cook, 1986) and France (Giraud, Bennejean, Guittet, \& Toquin, 1986). As the virus was spreading all over Europe and the clinical problems in poultry farms became increasingly serious, during the early 1990s (Cook et al. 1989a e 1989b) live attenuated vaccines were developed and became commercially available. By analysing strains circulating in Europe, Juhasz and Easton (1994) reported differences in the G gene between aMPV isolates and proposed the classification into A and B subtypes and confirmed the co-circulation of both subtypes. After this initial study, and due to the increasing use of sequence analysis, two further subtypes, named $\mathrm{C}$ and $\mathrm{D}$, were identified in France (Bayon-Auboyer, Arnauld, Toquin, \& Eterradossi, 2000; Toquin et al., 2006).

Despite these reports, molecular data on aMPV strains circulating in Europe are still poor and scattered (Catelli et al., 2004; Cecchinato et al., 2013a; Listorti et al., 2014; Franzo et al., 2017; Tucciarone et al., 
2017 a 2018; Ball, Forrester, \& Ganapathy, 2018; Andreopoulou et al., 2019), as most of them originate from few countries. In the last decades, subtype B has been generally the most frequently encountered subtype in Europe, although subtype A has been sporadically reported (Lupini et al., 2011).

In order to update the epidemiological picture of circulating strains, the present study was designed to molecularly characterize, by partial G gene sequencing, aMPV subtype B strains detected in Europe in the last years.

\section{Material and Methods}

\section{Sample collection}

The survey covered the time period from 2014 to 2019 and targeted aMPV subtype B detected during routine diagnostic activity performed in Italy by the Universities of Bologna and Padua or in Spain by the Centre de Sanitat Avícola de Catalunya i Aragó (CESAC).

Samples originated from different European countries and were usually collected during outbreaks of respiratory disorders referable to aMPV infections in turkey, chicken or guinea fowl flocks. Sampling was also performed for epidemiological purposes in absence of clear clinical signs.

As a rule, samples consisted of pools of 10 rhino-pharyngeal swabs per flock as previously suggested (Catelli et al., 2004).

\section{RNA extraction and PCR analysis}

RNA was extracted from each pool of swabs and subjected to a nested RT-PCR targeting the G gene (Cavanagh, Mawditt, Britton, \& Naylor, 1999) which allows the simultaneous detection and differentiation of subtypes A and B. Depending on the laboratory, this step was preceded by a multiplex real-time RT-PCR (qRT-PCR) screening test targeting the SH gene (Cecchinato et al., 2013b) and only positive samples were subsequently amplified by RT-nested PCR.

\section{Partial G gene sequencing}

The partial G gene amplicons obtained were purified using ExoSAP-ITExpress PCR Product Cleanup (Thermo Fisher Scientific, Massachusetts, USA) according to the manufacturer's instructions and sequenced in both directions using RT-nested PCR primers G5- (5'- CAAAGAGCCAATAAGCCCA-3') and G9+B (5'TAGTCCTCAAGCAAGTCCTC-3'), by a commercial sequencing service (Macrogen Europe, Amsterdam, The Netherlands). The obtained sequences were named using the following nomenclature: aMPV/B/Country of origin/Host species (Turkey: Ty, Chicken: Ck or Guinea fowl)/sample ID number/year of detection.

\section{Sequence and phylogenetic analysis}

Nucleotide sequences were edited and assembled using BioEdit software, then, using Clustal W, aligned against and compared with $\mathrm{G}$ gene sequences of:

- The most commonly used subtype B vaccines (i.e. aMPV/B vaccine - strain $\mathrm{VCO} 3$; aMPV/B vaccine Strain 11/94; aMPV/B vaccine - strain 1062 and aMPV/B vaccine - strain PL21)

- 82 already available European aMPV B sequences (Table S1)

- 59 extra-European aMPV-B sequences retrieved from GenBank(Table S2).

Phylogenetic relationships among the aMPV-B strains were reconstructed using the neighbor-joining algorithm implemented in MEGA X (Kumar et al., 2018). The branch support was calculated by performing 1000 bootstrap replicates; only branches supported by bootstrap values [?] $70 \%$ were considered reliable. Complete deletion option was selected before the analysis begins, to remove sites containing missing data or alignment gaps. Within-group mean pairwise genetic p-distance was estimated using MEGA X in order to evaluate the genetic heterogeneity of aMPV population.

\section{Results}


116 samples tested aMPV positive, all belonging to subtype B (n. 27 from France, n. 18 from Italy, n. 27 from Romania, n. 36 from Spain and n. 8 from the United Kingdom) and 116 partial G gene sequences were obtained. Sequence data have been submitted to the GenBank database under accession numbers MT432826-MT432923 and MT436220-MT436237 (Tables S3 to S7).

Phylogenetic analysis was carried out by reconstructing both a comprehensive tree (Figure 1) and five Country- specific trees (Figures S1 to S5).

Furthermore, a phylogenetic tree including selected European and extra-European aMPV-B sequences was generated (Figure 2).

Regardless of the Country of origin, aMPV strains detected from 2001 to 2019, herein referred to as "recent strains", were distinguished from those clustering with vaccines and field strains detected prior to the 1990s, referred to as "older strains" (from which the vaccines were established by attenuation) and those forming independent clades.

As a rule, aMPV strains were referred to as "vaccine-derived strains" if the maximum nucleotide sequence identity with a reference vaccine strain was greater than or equal to $99 \%$ and if they fell into the same phylogenetic cluster. The remaining strains were classified as "field strains".

On the above basis, 70 out of 116 aMPV-B strains were labelled as field strains (60\%); the remaining 46 strains $(40 \%)$ were identified as vaccine-derived strains.

A certain degree of geographic clustering was observed among Romanian, Spanish, French and Italian field strains (Figure 1). Some Italian strains also clustered together with some recent Greek strains.

French aMPV field strains (Figure S1) were divided in two clusters. The largest cluster displayed a withingroup mean p-distance of 0.004 and included chicken, turkey and guinea fowl strains detected from 2017 to 2019, while the smallest had a within-group mean p-distance of 0.000 and contains turkey strains detected in 2018. Vaccine-derived strains clustered respectively with vaccine strain PL21, 11/94 or VCO3 and were mostly detected in homologous-vaccinated turkeys or chickens, but also in unvaccinated guinea fowls.

Italian field strains were grouped in two main clusters (Figure S2): the larger one was composed of several sub-clusters including field strains detected from 2010 to 2019 in turkey, chicken, and guinea fowl flocks and showed a within-group mean p-distance of 0.011. The smaller one included field strains detected from 2001 to 2009 and in 2016 in turkeys and chickens, with a within-group mean p-distance of 0.004 . Vaccine-derived strains clustering with aMPV-B vaccine strains VCO3 or 1062 were detected from vaccinated turkeys.

Romanian aMPV-B field strains (Figure S3), detected from turkeys or chickens, fell within a single phylogenetic group showing a within-group mean p-distance of 0.001 . The vaccine-derived strains, all detected in vaccinated turkey flocks, clustered with vaccine strains PL21, 11/94 or VCO3.

In the Spanish tree (Figure S4) field strains fell within two main clusters: a larger one (within-group mean pdistance: 0.006) containing strains detected from 2014 to 2017; a smaller one (within-group mean p-distance: 0.003) containing viruses detected from 2014 to 2015. Spanish vaccine-derived strains clustered with vaccine strain 11/94 or PL21.

The British phylogenetic tree (Figure S5) exclusively displayed vaccine-derived strains, clustering with vaccine strain PL21, 11/94 or VCO3.

The tree reconstructed with representative aMPV-B sequences from all over the world (Figure 2) confirmed the Country-specific clustering trend seen for the European strains. Well-defined clusters were identified for field strains circulating in Brazil, Iran, Israel and Turkey. Vaccines, vaccine-derived and older strains clustered together and were showed in the figure compressed in two subtrees.

\section{Discussion}


In the present study, one hundred and sixteen aMPV-B partial G gene sequences, obtained during routine diagnostics in different European Countries in the last few years, were analysed by sequence and phylogenetic analyses in order to molecularly characterize them.

The G gene, which harbours mutations at variable positions between aMPV-B strains, was conveniently amplified by the routine diagnostic RT-PCR protocol employed in the study, and its sequencing proved useful for epidemiological purposes. Furthermore, its variability can give an indication of whether vaccine or field strains are present, making the differentiation relatively easy and cheap to perform.

Live attenuated aMPV vaccines are widely administered to prevent disease in turkeys and chickens, and the recovery of vaccine-derived strains is not unusual both in vaccinated or in unvaccinated flocks (Banet-Noach et al., 2009; Lupini et al., 2011; Chacon et al., 2011; Cecchinato et al., 2013a; Listorti et al., 2014; Arafa et al., 2015; Bayraktar et al., 2019; Andreopoulou et al., 2019).

The $40 \%$ of the aMPV-B strains detected in the present study were classified as vaccine-derived strains, being phylogenetically related and showing high nucleotide identity with live commercial vaccine strains licensed in Europe. As expected, vaccine-derived strains formed separate clusters depending on the vaccine strain of origin and were detected in all tested European countries.

A large part of the vaccine-derived strains analysed in the present study was detected in homologousvaccinated birds, from approximately two to four weeks after vaccination, and only occasionally in unvaccinated birds. Reversion to virulence of aMPV subtype A or B has been previously demonstrated (Catelli et al., 2006; Brown et al., 2011, Cecchinato et al., 2014). Therefore, the detection of vaccine-derived strains closely related to the applied vaccine, concurrently with respiratory signs, could be reliably linked to the vaccine reversion to virulence.

The detection of the strain aMPV/B/France/GuineaFowl/1060/18 in unvaccinated guinea fowls is noteworthy, since its partial G gene sequence shared $100 \%$ nucleotide identity with the vaccine strain $11 / 94$. It could be speculated that the vaccine virus could had been introduced as a contaminant by personnel, fomites, vehicles movement or airborne from neighbouring premises. The field veterinarian reported the presence of a turkey farm at approximately 500 meters from the guinea fowl one. Vaccinal strain 11/94 was applied in the turkey flock and the use of the litter from the turkey farm for fertilization of the surrounding crops was reported. The ability of vaccine-derived aMPVs to spread beyond the administration site is well known and it has been proven by Lupini et al. (2011) following a turkey rhinotracheitis outbreak caused by aMPV subtype A in unvaccinated turkeys.

The remaining $60 \%$ aMPV-B viruses analysed in the present study were classified as field strains since they clustered separately and showed a low nucleotide identity with vaccines and vaccine-derived strains.

The phylogenetic tree reconstructed with European sequences showed that the virus has continued to evolve from its first appearance in the '80s. In fact, more recently detected field strains belonged to clades phylogenetically distant from the older field strains, confirming the previously-reported aMPV tendency to evolve over time (Cecchinato et al., 2010).

Unlike vaccine-derived strains, field strains tended to cluster according to their geographic origin, with few exceptions. Distinct clusters were observed for French, Italian, Romanian and Spanish strains, yet some Italian and Greek field isolates clustered closely together, indicating a potential transmission route between these two countries (Tucciarone et al., 2017; Andreopoulou et al., 2019).

The molecular epidemiology of aMPV within each country was analysed in detail reconstructing countryrelated phylogenetic trees. Heterogeneous field strain populations seemed to co-exist within single European countries, with the only exception of Romania, where all identified strains were part of just one clade. This last finding could be explained by keeping into account that all the processed samples came from different sites within the same company in which viral circulation could be compartmentalized (Franzo et al., 2020).

French, Italian, and Spanish trees showed a rather heterogeneous field strain population, as the strains fell 
into more than one cluster, divided in several sub-clusters. The heterogeneity was further deduced from the within-group mean p-distance values. The highest value, indicating the highest heterogeneity of nucleotide sequence, was observed for Italian field strains, presumably because of the larger number of sequences available over a broader time period and coming from different commercial poultry companies.

Furthermore, time-related clustering was visible for Italian and Spanish field strains, as strains detected in the last few years tend to form separate clusters or sub-clusters.

As a common finding, recent field aMPV-B strains, belonging to different and distant phylogenetic clades, were detected in the presence of respiratory signs in vaccinated flocks. Due to the $\mathrm{G}$ gene variability observed in recent aMPV-B strains, and knowing that the encoded surface G glycoprotein is a key antigen for vaccine-induced immune protection (Naylor et al., 2007) and can evolve in order to avoid vaccine-induced immunity (Catelli et al., 2010; Cecchinato et al., 2010), re-assessment of protection conferred by commercially available vaccines against currently circulating aMPV field strains might be necessary to improve disease control strategies.

The obtained phylogenetic data revealed that aMPV-B strains clustered together irrespective of the host species where the viruses had been detected. A recent experimental challenge study showed that both chickens and turkeys are susceptible to aMPV-B infection with the same virus isolate (Brown et al., 2019). Moreover Cecchinato et al. (2018) reported an outbreak of respiratory disease in guinea fowls caused by aMPV-B strain identical to the ones circulating in the surrounding turkey flocks. Therefore, no evidences are currently available to support a host-specific adaptation of aMPV variants.

As a final remark, the tree reconstructed on European and extra-European strains further confirmed the geographic clustering of field strains. As a rule, field strains grouped together for country location and time period, suggesting a local evolution tendency of the virus that might have taken place after a single introduction event. The majority of recently detected European field strains were located in a big subtree indicating a certain genetic similarity and supporting the wide circulation of a quite homogeneous aMPV subtype B clade in European countries, with the only exception of two clusters of phylogenetically-distant French and Spanish strains.

In conclusion, the molecular characterization of aMPV subtype B and the differentiation between vaccines and field strains through $\mathrm{G}$ gene sequence analysis can be a useful tool towards a correct diagnosis and should be routinely applied in order to better address the control strategies. In this respect, current vaccine research is focused on reducing the issues connected to live attenuated vaccine reversion to virulence or the selection of potentially virulent subpopulations (Franzo et al., 2015). Therefore, considering the associated risks, further efforts should be directed at improving administration and biosecurity measures, in order to reduce their prolonged circulation and spreading.

Several research groups have attempted to develop efficacious, more stable and safer next-generation vaccines (Qingzhong et al., 1994; Tarpey et al., 2001; Kapczynski and Sellers, 2003; Kapczynski, 2004; Chary, Njenga, \& Sharma, 2005; Yu et al., 2013; Hu, Roth, Zsak, \& Yu, 2017) but, despite this effort, live vaccines still provide the greatest protection after homologous challenge.

Promising reverse genetics systems for aMPV have been developed in recent years for A, B and C subtypes (Naylor et al., 2004; Yu et al., 2010; Laconi et al., 2016) other than being an exceptional tool for the study of the virus properties (Brown et al., 2011), can be used for the development of rationally modified aMPV vaccines (Naylor, Lupini, \& Brown, 2010) or recombinant vaccines (Falchieri et al., 2013) expressing foreign genes.

\section{Conflict of Interest Statement}

The authors declare no conflict of interest.

\section{Ethical approval}


The authors confirm that the ethical policies of the journal, as noted on the journal's author guidelines page, have been adhered to. No ethical approval was required as the original research data of this article was obtained from oro-pharyngeal swabs collected for routine diagnostics purpose by field veterinarians.

\section{Data Availability Statement}

The data that support the findings of this study are available from the corresponding author upon reasonable request.

\section{References}

Andreopoulou, M., Franzo, G., Tucciarone, C. M., Prentza, Z., Koutoulis, K. C., Cecchinato, M., \& Chaligianni, I. (2019). Molecular epidemiology of infectious bronchitis virus and avian metapneumovirus in Greece.Poultry Science, 98(11), 5374-5384. https://doi.org/10.3382/ps/pez360 Arafa, A.-S., Mady, W., Hussein, A., Tamam, S., \& Madbouly, H. (2015). Molecular Characterization of VaccineDerived Mutants of Avian Meta-Pneumoviruses Isolated from Turkeys in Egypt. American Journal of Virology, 4(1), 1-11. https://doi.org/10.3844/ajvsp.2015.1.11 Ball, C., Forrester, A., \& Ganapathy, K. (2018). Co-circulation of genetically diverse population of vaccine related and unrelated respiratory mycoplasmas and viruses in UK poultry flocks with health or production problems. Veterinary Microbiology, 225, 132-138. https://doi.org/10.1016/j.vetmic.2018.09.009 Banet-Noach, C., Simanov, L., Laham-Karam, N., Perk, S., \& Bacharach, E. (2009). Longitudinal survey of avian metapneumoviruses in poultry in Israel: infiltration of field strains into vaccinated flocks.Avian Diseases, 53(2), 184-189.https://doi.org/10.1637/8466-090408-Reg.1Bayon-Auboyer, M. H., Arnauld, C., Toquin, D., \& Eterradossi, N. (2000). Nucleotide sequences of the F, L and G protein genes of two non-A/non-B avian pneumoviruses (APV) reveal a novel APV subgroup. Journal of General Virology, 81(11), 2723-2733. https://doi.org/10.1099/0022-1317-81-11-2723 Bayraktar, E., Umar, S., Yilmaz, A., Turan, N., Franzo, G., Tucciarone, C. M., ... Yilmaz, H. (2019). First Molecular Characterization of Avian Metapneumovirus (aMPV) in Turkish Broiler Flocks. Avian Diseases, 62(4), 425-430.https://doi.org/10.1637/11915-061818resnote.1Brown, P.A., Allee, C., Courtillon, C., Szerman, N., Lemaitre, E., Toquin, D., Mangart, J.M., ... Eterradossi, N. (2019). Host specificity of avian metapneumoviruses. Avian Pathology, 48(4), 311318.https://doi.org/10.1080/03079457.2019.1584390Brown, P. A., Lupini, C., Catelli, E., Clubbe, J., Ricchizzi, E., \& Naylor, C. J. (2011). A single polymerase (L) mutation in avian metapneumovirus increased virulence and partially maintained virus viability at an elevated temperature. Journal of General Virology, 92(Pt 2), 346-354. https://doi.org/10.1099/vir.0.026740-0 Catelli, E., De Marco, M. A., Delogu, M., Terregino, C., \& Guberti, V. (2001). Serological evidence of avian pneumovirus infection in reared and free-living pheasants. Veterinary Record, 149(2), 56-58. https://doi.org/10.1136/vr.149.2.56 Catelli, E., Cecchinato, M., Cassandro, M., Delogu, M., De Matteo, P., Franciosi, C., .. John Naylor, C. (2004). Avian Pneumovirus infection in turkey and broiler farms in Italy: a virological, molecular and serological field survey. Italian Journal of Animal Science, 3(3), 287-292. https://doi.org/10.4081/ijas.2004.287

Catelli, E., Cecchinato, M., Savage, C. E., Jones, R. C., \& Naylor, C. J. (2006). Demonstration of loss of attenuation and extended field persistence of a live avian metapneumovirus vaccine. Vaccine, 24(42-43), 6476-6482. https://doi.org/10.1016/j.vaccine.2006.06.076 Cavanagh, D., Mawditt, K., Britton, P., \& Naylor, C. J. (1999). Longitudinal field studies of infectious bronchitis virus and avian pneumovirus in broilers using type-specific polymerase chain reactions.Avian Pathology, 28(6), 593-605. https://doi.org/10.1080/03079459994399 Cecchinato, M., Catelli, E., Lupini, C., Ricchizzi, E., Prosperi, S., \& Naylor, C. J. (2014). Reversion to virulence of a subtype B avian metapneumovirus vaccine: Is it time for regulators to require availability of vaccine progenitors? Vaccine, 32(36), 4660-4664. https://doi.org/10.1016/j.vaccine.2014.06.030 Cecchinato, M., Ferreira, H. L., Munir, M., \& Catelli, E. (2017). Avian metapneumovirus. In Mononegaviruses of veterinary importance. Volume 2: molecular epidemiology and control. https://doi.org/10.1079/9781780644172.0127 Cecchinato, M., Lupini, C., Silveira, F., Listorti, V., Mescolini, G., Morandini, E., .. Catelli, E. (2018). Molecular characterization of avian metapneumovirus from Guinea fowls (numida meleagridis).Pakistan Veterinary Journal, 38(4), 419423. https://doi.org/10.29261/pakvetj/2018.088 Cecchinato, M, Drigo, M., Lupini, C., Martini, M., Listorti, 
V., Franzo, G., .. Catelli, E. (2013a). Field survey of avian metapneumovirus in Northern Italy. Large Animal Review, 19(6), 267-270. Cecchinato, M., Catelli, E., Lupini, C., Ricchizzi, E., Clubbe, J., Battilani, M., \& Naylor, C. J. (2010). Avian metapneumovirus (AMPV) attachment protein involvement in probable virus evolution concurrent with mass live vaccine introduction. Veterinary Microbiology, 146(1-2), 24-34. https://doi.org/10.1016/j.vetmic.2010.04.014 Cecchinato, M., Lupini, C., Munoz Pogoreltseva, O. S., Listorti, V., Mondin, A., Drigo, M., \& Catelli, E. (2013b). Development of a real-time RT-PCR assay for the simultaneous identification, quantitation and differentiation of avian metapneumovirus subtypes A and B.Avian Pathology, 42(3), 283-289. https://doi.org/10.1080/03079457.2013.788130 Cecchinato, M., Lupini, C., Ricchizzi, E., Falchieri, M., Meini, A., Jones, R. C., \& Catelli, E. (2012). Italian Field Survey Reveals a High Diffusion of Avian Metapneumovirus Subtype B in Layers and Weaknesses in the Vaccination Strategy Applied. Avian Diseases, 56(4), 720-724. https://doi.org/10.1637/10202-041312-reg.1 Chacon, J. L., Mizuma, M., Vejarano, M. P., Toquin, D., Eterradossi, N., Patnayak, D. P., .. Piantino Ferreira, A. J. (2011). Avian Metapneumovirus Subtypes Circulating in Brazilian Vaccinated and Nonvaccinated Chicken and Turkey Farms. Avian Diseases, 55(1), 82-89. https://doi.org/10.1637/9501-081310-reg.1 Chary, P., Njenga, M. K., \& Sharma, J. M. (2005). Protection by recombinant viral proteins against a respiratory challenge with virulent avian metapneumovirus. Veterinary Immunology and Immunopathology, 108(3-4), 427432.https://doi.org/10.1016/j.vetimm.2005.06.012Cook, J.K.A., Ellis, M.M., Dolby, C.A., Huggins, M.B., Holmes, H.C., Finney, P.M. (1989a). A Live Attenuated Turkey Rhinotracheitis Virus Vaccine. 1. Stability of the Attenuated Strain. Avian Pathology, 18(3), 511-522. https://doi.org/10.1080/03079458908418623 Cook, J. K. A., Dolby, C. A., Ellis, M. M., Huggins, M. B., Holmes, H. C., \& Finney, P. M. (1989b). A Live Attenuated Turkey Rhinotracheitis Virus Vaccine. 2. the Use of the Attenuated Strain as an Experimental Vaccine. Avian Pathology, 18(3), 523-534. https://doi.org/10.1080/03079458908418624 Falchieri, M., Lupini, C., Cecchinato, M., Catelli, E., Kontolaimou, M., \& Naylor, C. J. (2013). Avian metapneumoviruses expressing Infectious Bronchitis virus genes are stable and induce protection. Vaccine, 31(22), 2565-2571. https://doi.org/10.1016/j.vaccine.2013.03.055 Franzo, G., Naylor, C. J., Drigo, M., Croville, G., Ducatez, M. F., Catelli, E., ... Cecchinato, M. (2015). Subpopulations in aMPV vaccines are unlikely to be the only cause of reversion to virulence. Vaccine, 33(21), 2438-2441. https://doi.org/10.1016/j.vaccine.2015.03.092 Franzo, G., Tucciarone, C. M., Enache, M., Bejan, V., Ramon, G., Koutoulis, K. C., \& Cecchinato, M. (2017). First Report of Avian Metapneumovirus Subtype B Field Strain in a Romanian Broiler Flock During an Outbreak of Respiratory Disease. Avian Diseases, 61(2), 250-254.https://doi.org/10.1637/11557121216-ResNote.1Franzo, G., Tucciarone, C.M., Moreno, A., Legnardi, M., Massi, P., Tosi, G., ..., Cecchinato, M. (2020). Phylodynamic analysis and evaluation of the balance between anthropic and environmental factors affecting IBV spreading among Italian poultry farms. Scientific Reports, 10, 7289. https://doi.org/10.1038/s41598-020-64477-4 Giovanardi, D., Lupini, C., Pesente, P., Rossi, G., Ortali, G., \& Catelli, E. (2014). Longitudinal field studies of Avian metapneumovirus and Turkey hemorrhagic enteritis virus in turkeys suffering from colibacillosis associated mortality. Veterinary Research Communications, 38(2), 129-137. https://doi.org/10.1007/s11259-014-9596-z Giraud, P., Bennejean, G., Guittet, M., \& Toquin, D. (1986). Turkey rhinotracheitis in France: preliminary investigations on a ciliostatic virus. Veterinary Record, 119(24), 606-607. https://doi.org/10.1136/vr.119.24.606-a Hu, H., Roth, J. P., Zsak, L., \& Yu, Q. (2017). Engineered Newcastle disease virus expressing the F and G proteins of AMPV-C confers protection against challenges in turkeys. Scientific Reports, 7(1), 4025. https://doi.org/10.1038/s41598017-04267-7 Juhasz, K., \& Easton, A. J. (1994). Extensive sequence variation in the attachment (G) protein gene of avian pneumovirus: Evidence for two distinct subgroups. Journal of General Virology, 75 (11), 2873-2880. https://doi.org/10.1099/0022-1317-75-11-2873 Kapczynski, D. R. (2004). Development of a Virosome Vaccine Against Avian Metapneumovirus Subtype C for Protection in Turkeys. Avian Diseases, 48(2), 332-343. https://doi.org/10.1637/7115 Kapczynski, D. R., \& Sellers, H. S. (2003). Immunization of Turkeys with a DNA Vaccine Expressing Either the F or N Gene of Avian Metapneumovirus. Avian Diseases, 47(4), 1376-1383. https://doi.org/10.1637/7033 Kumar, S., Stecher, G., Li, M., Knyaz, C., \& Tamura, K. (2018). MEGA X: Molecular evolutionary genetics analysis across computing platforms.Molecular Biology and Evolution, 35(6), 1547-1549. https://doi.org/10.1093/molbev/msy096 Laconi, A., Clubbe, J., Falchieri, M., Lupini, C., Cecchinato, M., Catelli, E., ... Naylor, C. J. (2016). A comparison 
of AMPV subtypes A and B full genomes, gene transcripts and proteins led to reverse-genetics systems rescuing both subtypes. Journal of General Virology, 97(6), 1324-1332. https://doi.org/10.1099/jgv.0.000450 Listorti, V., Lupini, C., Cecchinato, M., Pesente, P., Rossi, G., Giovanardi, D., .. Catelli, E. (2014). Rapid detection of subtype B avian metapneumoviruses using RT-PCR restriction endonuclease digestion indicates field circulation of vaccine-derived viruses in older turkeys. Avian Pathology, 43(1), 51-56. https://doi.org/10.1080/03079457.2013.866212 Lupini, C., Cecchinato, M., Ricchizzi, E., Naylor, C. J., \& Catelli, E. (2011). A turkey rhinotracheitis outbreak caused by the environmental spread of a vaccine-derived avian metapneumovirus. Avian Pathology, 40(5), 525-530. https://doi.org/10.1080/03079457.2011.607428 McDougall, J. S., \& Cook, J. K. (1986). Turkey rhinotracheitis: preliminary investigations. The Veterinary Record, 118(8), 206-207. https://doi.org/10.1136/vr.118.8.206 Naylor, C. J., Brown, P. A., Edworthy, N., Ling, R., Jones, R. C., Savage, C. E., \& Easton, A. J. (2004). Development of a reverse-genetics system for Avian pneumovirus demonstrates that the small hydrophobic $(\mathrm{SH})$ and attachment $(\mathrm{G})$ genes are not essential for virus viability. Journal of General Virology, 85 (Pt 11), 3219-3227. https://doi.org/10.1099/vir.0.80229-0 Naylor, C. J., Ling, R., Edworthy, N., Savage, C. E., \& Easton, A. J. (2007). Avian metapneumovirus SH gene end and $\mathrm{G}$ protein mutations influence the level of protection of live-vaccine candidates.Journal of General Virology, 88 (Pt 6), 1767-1775. https://doi.org/10.1099/vir.0.82755-0 Naylor, C. J., Lupini, C., \& Brown, P. A. (2010). Charged amino acids in the AMPV fusion protein have more influence on induced protection than deletion of the SH or G genes. Vaccine, 28 (41), 6800-6807. https://doi.org/10.1016/j.vaccine.2010.07.015 Qingzhong, Y., Barrett, T., Brown, T. D. K., Cook, J. K. A., Green, P., Skinner, M. A., \& Cavanagh, D. (1994). Protection against turkey rhinotracheitis pneumovirus (TRTV) induced by a fowlpox virus recombinant expressing the TRTV fusion glycoprotein (F). Vaccine, 12(6), 569-573. https://doi.org/10.1016/0264410X(94)90319-0 Tarpey, I., Huggins, M. B., Davis, P. J., Shilleto, R., Orbell, S. J., \& Cook, J. K. A. (2001). Cloning, expression and immunogenicity of the avian pneumovirus (Colorado isolate) F protein. Avian Pathology, 30(5), 471-474.https://doi.org/10.1080/03079450120078653Toquin, D., Bayon-Auboyer, M.H., Eterradossi, N. \& Jestin, V. (1999). Isolation of a pneumovirus from a Muscovy duck. The Veterinary Record, 23, 680. Toquin, D., Guionie, O., Jestin, V., Zwingelstein, F., Allee, C., \& Eterradossi, N. (2006). European and American subgroup $\mathrm{C}$ isolates of avian metapneumovirus belong to different genetic lineages. Virus Genes, 32(1), 97-103. https://doi.org/10.1007/s11262-005-5850-3 Tucciarone, C.M., Andreopoulou, M., Franzo, G., Prentza, Z., Chaligiannis, I., \& Cecchinato, M. (2017). First Identification and Molecular Characterization of Avian metapneumovirus Subtype B from Chickens in Greece. Avian Diseases, 61(3), 409-413. https://doi.org/10.1637/11631-032017-CaseR Tucciarone, C. M., Franzo, G., Lupini, C., Alejo, C. T., Listorti, V., Mescolini, G., ... Cecchinato, M. (2018). Avian Metapneumovirus circulation in Italian broiler farms. Poultry Science, 97(2), 503-509. https://doi.org/10.3382/ps/pex350 Yu, Q., Estevez, C., Song, M., Kapczynski, D., \& Zsak, L. (2010). Generation and biological assessment of recombinant avian metapneumovirus subgroup $\mathrm{C}$ (aMPV-C) viruses containing different length of the $\mathrm{G}$ gene. Virus Research, 147(2), 182-188. https://doi.org/10.1016/j.virusres.2009.10.021 Yu, Q., Roth, J. P., Hu, H., Estevez, C. N., Zhao, W., \& Zsak, L. (2013). Protection by Recombinant Newcastle Disease Viruses (NDV) Expressing the Glycoprotein (G) of Avian Metapneumovirus (aMPV) Subtype A or B against Challenge with Virulent NDV and aMPV. World Journal of Vaccines, 03(04), 130-139.https://doi.org/10.4236/wjv.2013.34018

\section{Figure legends}

Figure 1. Phylogenetic tree based on G gene nucleotide sequences of 116 European aMPV-B strains detected in the present study, including the most commonly used subtype B vaccines and 82 European aMPV-B strains, previously obtained or retrieved from GenBank. The two subtrees containing vaccines and vaccine-derived strains have been compressed. Field aMPV-B strains clusters are included in square brackets and coloured by country of origin. Only bootstrap values [?] 70 are reported.

Figure 2. Phylogenetic tree based on G gene nucleotide sequences of selected European and extra-European aMPV-B strains, previously obtained or retrieved from GenBank. The two subtrees containing vaccines and vaccine-derived strains have been compressed. Field aMPV-B strains clusters are coloured by country of origin. The country of origin was added in brackets next to the strain name when it was not clearly specified in the very name. Only bootstrap values [?] 70 are reported. 


\section{Supplementary figure legends}

Figure S1. Phylogenetic tree based on G gene nucleotide sequences of 27 French aMPV-B strains detected in the present study, including the most commonly used subtype B vaccines (marked with a green triangle) and 2 French aMPV-B strains previously obtained. Field aMPV-B strains clusters are included in square brackets. Only bootstrap values [?] 70 are reported.

Figure S2. Phylogenetic tree based on G gene nucleotide sequences of 18 Italian aMPV-B strains detected in the present study, including the most commonly used subtype B vaccines (marked with a green triangle) and 42 Italian aMPV -B strains, previously obtained or retrieved from GenBank. Field aMPV-B strains clusters are included in square brackets. Only bootstrap values [?] 70 are reported.

Figure S3. Phylogenetic tree based on G gene nucleotide sequences of 27 Romanian aMPV-B strains detected in the present study and the most commonly used subtype B vaccines (marked with a green triangle). Field aMPV-B strains clusters are included in square brackets. Only bootstrap values [?] 70 are reported.

Figure S4. Phylogenetic tree based on G gene nucleotide sequences of 36 Spanish aMPV-B strains detected in the present study and the most commonly used subtype B vaccines (marked with a green triangle). Field aMPV-B strains clusters are included in square brackets. Only bootstrap values [?] 70 are reported.

Figure S5. Phylogenetic tree based on G gene nucleotide sequences of 8 British aMPV-B strains detected in the present study, including the most commonly used subtype B vaccines (marked with a green triangle) and 10 British aMPV -B strains, previously obtained or retrieved from GenBank. Only bootstrap values [?] 70 are reported. 
Figure 1

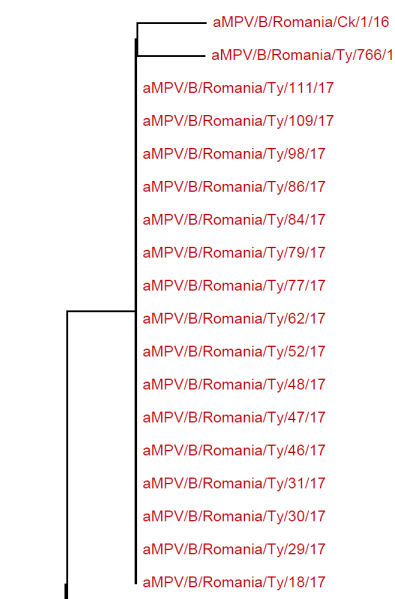

7

aMPV/B/Spain/Ck/11929/17

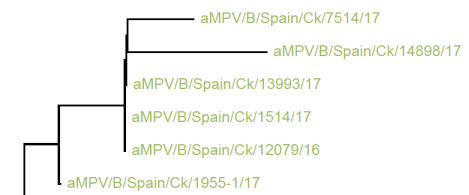

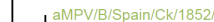

aMPV/B/Spain/CK/2191/16

aMPV/B/Spain/Ck/434/17

aMPV/B/Spain/Ck/690/17

aMPV/B/Spain/Ck/691/17

aMPV/B/Spain/Ck/16563/17

aMPV/B/Spain/Ck/6414/17

aMPV/B/Spain/Ck/6413/17

aMPV/B/Spain/Ck/3192/17

aMPV/B/Spain/Ck/570-6/17

aMPV/B/Spain/Ck/12399/16

aMPV/B/Spain/Ck/10283-2/16

aMPV/B/Spain/Ty/1255/16

aMPV/B/Spain/Ty/2598/15

aMPV/B/Spain/Ck/1080-1/15

aMPV/B/Spain/Ty/11776/14

31aMPV Chicken Greece 2016

33ampV Chicken Greece 2010

aMPV/B/ttaly/GuineaFow/1818/12

aMPV/B/taly/Ty/2009/13

aMPV/B/taly/Ck/569-03/15

aMPV/B/Italy/Ck/570-02/15

aMPV/B/taly/Ck/575-03/15

aMPV/B/ltaly/Ck/575-04/15

aMPV/B/taly/Ty/629-04/16

_ aMPV/B/ttaly/Ty/1997/12

38aMPV Chicken Greece 2018

35amPV Chicken Greece 2016

29amPV Chicken Greece 2010

27aMPV Chicken Greece 2016

10aMPV Chicken Greece 2016

aMPV/B/taly/CK/164/13

IT/Ty/532/10

IT/Ty/531/10

aMPV/B/taly/Ck/91/14

aMPV/B/taly/CK/185/15

aMPV/B/ttaly/Ck/86/14

- aMPV/B/ttaly/Ck/248/15

aMPV/B/taly/Ck/478/16

aMPV/B/ttaly/Ck/509/16

aMPV/B/ltaly/Ck/541/16

aMPV/B/taly/Ck/556/16 11

aMPV/B/taly/Ty/626-10/16

aMPV/B/ttaly/Ty/627-10/16

aMPV/B/taly/Ck/1298/19

aMPV/B/taly/Ck/41/14

aMPV/B/ttaly/Ck/92/14

aMPV/B/ttaly/Ck/95/14

aMPV/B/ttaly/Ck/104/14 


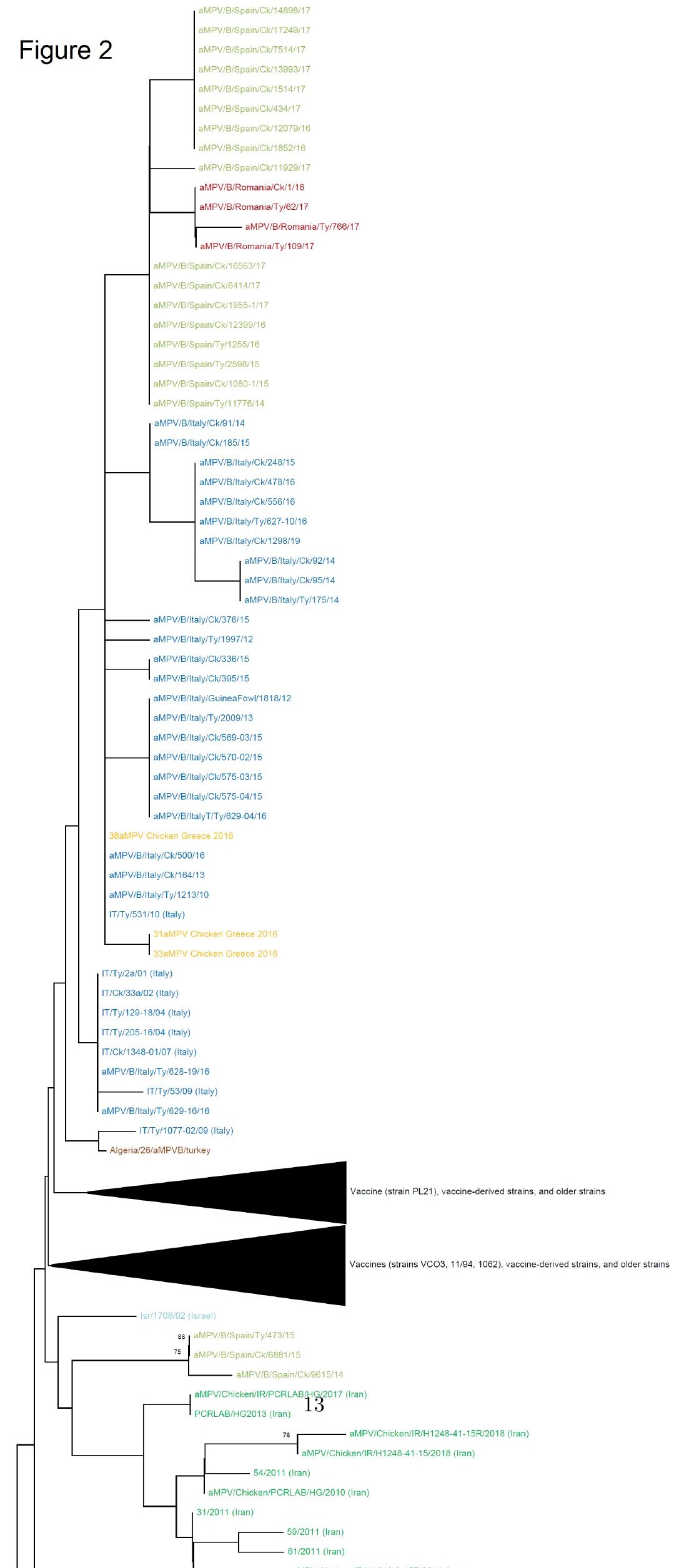

